\title{
Study of CD69 antigen expression and integrity of leukocyte cellular membrane in stored platelet concentrates following irradiation and treatment with Mirasol ${ }^{\circledR}$ PRT System
}

\author{
Elżbieta Lachert' A-F, Jolanta Woźniak ${ }^{2, A-F}$, Jolanta Antoniewicz-Papis ${ }^{1, A-F}$, Agnieszka Krzywdzińska 2, B, E, \\ Jolanta Kubis ${ }^{1}$, B-F , Agata Mikołowska ${ }^{1, B-F}$, Magdalena Letowska, ${ }^{1, B-F}$ \\ ${ }^{1}$ Department of Transfusion Medicine, Institute of Hematology and Transfusion Medicine, Warszawa, Poland \\ ${ }^{2}$ Department of Diagnostics for Hematology, Institute of Hematology and Transfusion Medicine, Warszawa, Poland \\ A - research concept and design; $\mathrm{B}$ - collection and/or assembly of data; $\mathrm{C}$ - data analysis and interpretation; \\ $D$ - writing the article; $E$ - critical revision of the article; $F$ - final approval of article
}

Address for correspondence

Elżbieta Lachert

E-mail: elachert@ihit.waw.pl

\section{Funding sources}

none declared

Conflict of interest

none declared

Received on September 23, 2016

Revised on October 25, 2016

Accepted on January 05, 2017

DOI

10.17219/acem/68290

Copyright

Copyright by Author(s)

This is an article distributed under the terms of the

Creative Commons Attribution Non-Commercial License

(http://creativecommons.org/licenses/by-nc-nd/4.0/)

\begin{abstract}
Background. Leukocytes in transfused blood components, particularly residual lymphocytes, have been shown to contribute to the occurrence of various adverse reactions. One of the most severe is transfusionassociated graft versus host disease (TA-GvHD) following transfusion of blood components contaminated with immunocompetent $T$ lymphocytes. Irradiation is a routine method for protection against TA-GvHD. According to the literature, some pathogen reduction methods have also been proven effective for the inactivation of T lymphocytes, and so they may be considered as an alternative to irradiation.

Objectives. Comparison of CD69 antigen expression and the integrity of the leukocyte cellular membrane in stored platelet concentrates (PCS) following irradiation with the Gammacell 3000 Elan (Nordion Inc., Ottawa, Canada) and treatment with the Miraso ${ }^{\circledR}$ Pathogen Reduction Technology (PRT) System (Terumo BCT, Lakewood, USA).
\end{abstract}

Material and methods. The study included seven experiments. For each experiment we used 3 PCS, for Mirasol ${ }^{\circledR}$ PRT System treatment (M), for Gammacell 3000 Elan irradiation (R), and for the control (C). 7-amino-actinomycin D (7-AAD, Becton Dickinson, Franklin Lakes, USA) permeability was used to determine lymphocyte viability while CD69 antigen expression was the marker of lymphocyte activation. Analyses of 7-AAD and CD69 antigen expression were performed in a FACS Canto I flow cytometer (Becton Dickinson, USA).

Results. During 6 storage days, viable lymphocyte count decreased to $28 \%(p=0.001)$ in the Miraso ${ }^{\circledR}$ PRT System treated PCs and to $65 \%(p=0.004)$ in the irradiated PCs. A statistically significant increase in CD69 expression in the irradiated PCs was observed; 1.3-fold on day 3 and 1.5-fold on day 6 . In the Mirasol ${ }^{\circledR}$ PRT System treated PCs, no statistically significant increase was observed.

Conclusions. The in vitro results suggest that the Mirasol ${ }^{\circledR}$ PRT System is as effective as irradiation due to donor leukocyte inactivation capacity.

Key words: blood component, leukocyte viability, CD69 
Leukocytes in transfused blood components, particularly residual lymphocytes, have been shown to contribute to the occurrence of a variety of adverse reactions. A particularly serious post-transfusion complication/reaction is transfusion-associated graft versus host disease (TA-GvHD) following the transfusion of immunocompetent $\mathrm{T}$ lymphocytes, which the recipient's immunological system is incapable of destroying. TA-GvHD, although extremely rare (0.1-1\% of all serious adverse events and reactions according to Serious Hazards of Transfusion (SHOT)), is associated with a high mortality rate (80-90\%) due to lack of effective treatment. It is therefore a serious clinical challenge. ${ }^{1-3}$

To date, the only routine method used for the prevention of TA-GvHD in high-risk patients was transfusion of cellular blood components subjected to gamma irradiation $\left(\mathrm{Cs}^{137}, \mathrm{Co}^{60}\right)$ or X-ray. ${ }^{4,5}$ Advancement in pathogen inactivation methods has demonstrated that some inactivation methods induce irreversible changes in the nucleic acids of viruses, bacteria or protozoa by inhibiting their replication and have similar effect on leukocytes. It stands to reason that they may also prove effective for inactivation of T lymphocytes. This implies that some pathogen inactivation methods may be considered as an alternative to gamma irradiation. In some countries (France, Spain, Austria, Luxemburg), platelet concentrates (PCs) for high-risk patients are subjected exclusively to pathogen inactivation with the Mirasol ${ }^{\circledR}$ PRT System or the Intercept ${ }^{\mathrm{TM}}$ Blood System. ${ }^{6,7}$

In Poland the Mirasol ${ }^{\circledR}$ PRT System was introduced in 2009, first for fresh frozen plasma (FFP) then for PCs. The Mirasol ${ }^{\circledR}$ PRT System is based on exposure to riboflavin (vitamin B2) and UV-light. The process induces irreparable damage to nucleic acids and so inhibits the replication of pathogens and white blood cells/leukocytes. In this way, the infectivity of blood components is reduced and the recipient is protected against TA-GvHD. ${ }^{8,9}$ The efficacy of the leukocyte inactivation process, mononuclear $\mathrm{T}$ cells (MNCs) in particular, is determined in such in vitro studies as an evaluation of proliferation parameters, limiting dilution assay (LDA) or assessment of DNA modification. In use, however, there are also other tests that can serve as indirect measurement of leukocyte inactivation. Among others, these include: CD69 antigen expression and integrity of the leukocyte cellular membrane. The focus of the study was the comparison of CD69 antigen expression and integrity of leukocyte cellular membrane in stored platelet concentrates (PCs) following irradication with the Gammacel 3000Elan (Nordion, Canada) and treatment with the Mirasol ${ }^{\circledR}$ Pathogen Reduction Technology (PRT) System (Terumo BCT, USA). Comparison of CD69 antigen expression and integrity of leukocyte cellular membrane in stored platelet concentrates (PCs) following irradiation with the Gammacell 3000 Elan (Nordion, Canada) and treatment with the Mirasol ${ }^{\circledR}$ Pathogen Reduction Technology (PRT) System (Terumo BCT, USA).
CD69 antigen expression is an early marker of T lymphocyte inactivation. CD69 antigen expression also appears on B lymphocytes and macrophages and participates in transmission of the activating signal which leads to synthesis of various cytokines such as interleukin-2 (IL-2) and interferon- $\gamma($ IFN- $\gamma){ }^{10}$

Although the lack of $\mathrm{T}$ cell proliferative capacity confirmed in LDA as well as the inhibition of nucleic acid amplification are standard and well described evaluation methods for an assessment of lymphocyte inactivation, there are also other methods that can be considered additional parameters for measuring lymphocyte inactivation in the Mirasol ${ }^{\circledR}$ PRT System. These include: viability of lymphocytes (7-AAD) and integrity of leukocyte cellular membrane. ${ }^{11}$

The effect of inactivation was measured with flow cytometry by evaluation of lymphocyte survival with 7-AAD assay. 7-AAD is a fluorescent dye with strong DNA affinity which labels DNA in damaged, nonviable cells. Due to a lower wave-length and less intensive fluorescence, it is a useful alternative to propidium iodide (PI) and enables simultaneous staining with phycoerythrin (PE)-labeled antibodies. Although 7-AAD is not as bright as PI, nonviable cells can easily be distinguished from viable cells. ${ }^{12,13}$ The effect of lymphocyte inactivation can also be evaluated based on expression levels of lymphocyte activation markers. One of such markers is the CD69 surface molecule, a widely expressed leukocyte receptor with rapid kinetic onset after activation. It regulates the immunological system by modulating the expression of various cytokines and contributes to the regulation of type I and II interferon (IFN). It also plays an important role in T-cell homeostasis. CD69 has also been implicated in the development of autoimmune diseases. Despite extensive studies, the function of CD69 in the immune response against infective intracellular pathogens has not yet been elucidated. ${ }^{14}$

The focus of the study is to compare the effect of gamma-irradiation and the Mirasol ${ }^{\circledR}$ PRT System on PC lymphocyte viability and activation.

\section{Material and methods}

\section{Preparation of platelet concentrates}

The study included seven experiments. For each experiment we used 3 PCs for Mirasol ${ }^{\circledR}$ PRT System treatment (M), for Gammacell 3000 Elan irradiation (R), and for control (C). The same type of bag was used for storage of all of the PC units. The average PC volume was $234 \mathrm{~mL}$ for M, $227 \mathrm{~mL}$ for $\mathrm{R}$ and $236 \mathrm{~mL}$ for $\mathrm{C}$. The average platelet count per unit was $3.4 \times 10^{11}$ for $\mathrm{M}, 3.33 \times 10^{11}$ for $\mathrm{R}$ and $3.50 \times 10^{11}$ for $\mathrm{C}$. The average leukocyte count was 225 $\times 10^{6}$ for M, $204 \times 10^{6}$ for $\mathrm{R}$ and $212 \times 10^{6}$ for $\mathrm{C}$. The PCs were prepared according to Polish guidelines. ${ }^{15}$ 


\section{Gammacell 3000 Elan and Mirasol ${ }^{\circledR}$ PRT System}

The gamma irradiation source was a Gammacell 3000 Elan irradiator with radioactive ${ }^{137} \mathrm{Cs}$ (powder chloride caesium) in a double stainless steel capsule at an absorptive dose of $25 \mathrm{~Gy}^{*}$. It is the requirement of the quality assurance (QA) system that the distribution of absorbed dose is verified every three years and a leak test is performed annually. ${ }^{16}$ Pathogen inactivation was performed with the Mirasol ${ }^{\circledR}$ PRT System which is comprised of an illuminator and a set of disposable bags. After adding riboflavin ( $35 \mathrm{~mL}$ riboflavin: $500 \mu \mathrm{M}$ in $0.9 \%$ sodium chloride) to the PCs in the illumination bag, the component was placed in the illuminator and exposed to UV light for approximately 6-10 min. Time duration was automatically calculated by the system based on PC volume. Light energy was $6.24 \mathrm{~J} / \mathrm{mL}$. Following illumination, the PCs were stored for 6 days. ${ }^{17}$

The study procedure ran as follows; $35 \mathrm{~mL}$ of $500 \mu \mathrm{M}$ riboflavin solution was added to the PCs in group $\mathrm{M}, 35 \mathrm{~mL}$ of saline solution ( $0.9 \% \mathrm{NaCl}$, Ravimed, Poland) was added to the PCs in groups $\mathrm{R}$ and $\mathrm{C}$. The $\mathrm{M}$ group bags were immediately illuminated for about $6 \mathrm{~min}$ at $6.24 \mathrm{~J} / \mathrm{mL}$. At the same time, the $\mathrm{R}$ group bags were irradiated and put in storage for 6 days at $22^{\circ} \mathrm{C}$ with agitation (Helmer, Fresenius Kabi, USA) and the $\mathrm{C}$ group bags were put in storage for 6 days at $22^{\circ} \mathrm{C}$ with agitation. On days 1,3 and 6 , samples for flow cytometric analysis were collected from bags $M, R$ and $C$.

\section{Flow cytometry analysis}

Permeability to 7-AAD dye was used as the measure of lymphocyte survival rate while anti-CD69-APC staining (Becton Dickinson, Franklin Lakes, USA) was used to assess lymphocyte activation. PC samples were concurrently stained with anti-CD45-PE antibodies (Becton Dickinson, Franklin Lakes, USA) for identification and gating purposes. The fluorescence-labeled samples were analyzed by three-color analysis using a flow cytometer. Before each run, the cytometer was calibrated with Setup and Tracking beads (Becton Dickinson, Franklin Lakes, USA) and color-compensated with CaliBRITE beads for 4-color flow cytometer setup using CaliBRITE APC with CaliBRITE 3 (Becton Dickinson, Franklin Lakes, USA).

\section{Lymphocyte identification}

List-mode data files containing at least 1000 CD45+ lymphocytes were collected for subsequent offline analysis using FACSDiva software (Becton Dickinson, Frank-

\footnotetext{
* A gray is a derived unit of ionizing radiation dose in the International System of Units (SI). It is defined as the absorption of one joule of radiation energy per kilogram of matter.
}

lin Lakes, USA). CD45+ lymphocytes were identified by gating on CD45 bright fluorescence and low side scatter (Fig. 1A, blue gate).

\section{Lymphocyte viability}

Lymphocytes in the CD45+ gate were analyzed for 7-AAD fluorescence and displayed as dot plots (CD45 vs. 7-AAD). The proportion of unstained cells, not permeable to 7-AAD, (viable) and stained cells, 7-AAD permeable, (nonviable) was determined by setting the marker immediately to the right of the lymphocyte population in the untreated controls on day 1 after $\mathrm{PC}$ preparation (Fig. 1A, B). The population of nonviable cells fell in region Q2 (CD45+7-AAD+). The population of viable cells occupied region Q1 (CD45+7-AAD-).

\section{CD69 activation assay}

CD69 expression (unstimulated) was examined only for viable lymphocytes (CD45+7-AAD-) and displayed as dot plots CD69 vs. 7-AAD. To determine the number of activated lymphocytes, the marker was set by using the appropriate isotype control (mouse IgG1-APC, Becton Dickinson, Franklin Lakes, USA). The result was > 97.5\% of CD69 negative cells within the Q1 region. CD69 positive viable cells (activated) were located in region Q4-1 (CD69+7-AAD-) (Fig. 1A, C). The Mean Fluorescence Intensity (MFI) of CD69 was determined for all CD45+ and 7-AAD non-permeable cells.

\section{Statistical analysis}

All reported values were calculated with STATISTICA 10 (StatSoft Inc.) statistical software program. The differences between groups were evaluated by paired twotailed t-tests. P-values $<0.05$ were considered statistically significant.

\section{Results}

\section{Lymphocyte viability}

Permeability to 7-AAD staining was the criterion for differentiation between the two populations of cells: permeable-nonviable cells 7 -AAD $(+)$ and non-permeableviable cells 7 -AAD (-).

The gates for analysis were determined on day 1 using control samples (Fig. 1B). Immediately after gamma irradiation and Mirasol ${ }^{\circledR}$ PRT System treatment, the percentage of viable cells 7-AAD(-) on day 1 was approximately $99 \%$ in all tested samples (C, M and R). In all control samples, the number of viable cells did not decrease significantly during the 6 days of storage and was maintained at a level of over $96 \%$ (Fig. 2). Cell viability significantly decreased during storage in both the $\mathrm{R}$ and $\mathrm{M}$ treated sam- 


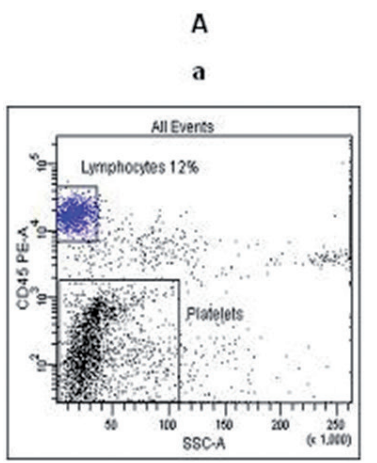

b

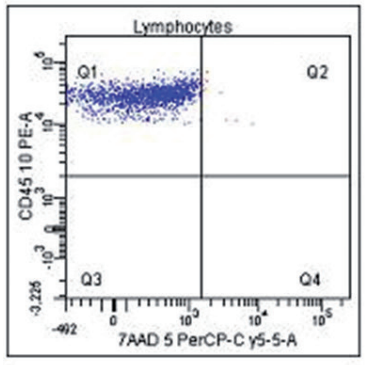

C

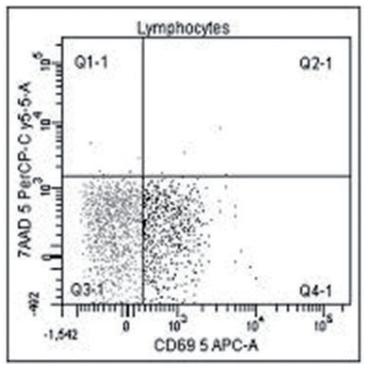

B

Control (C)
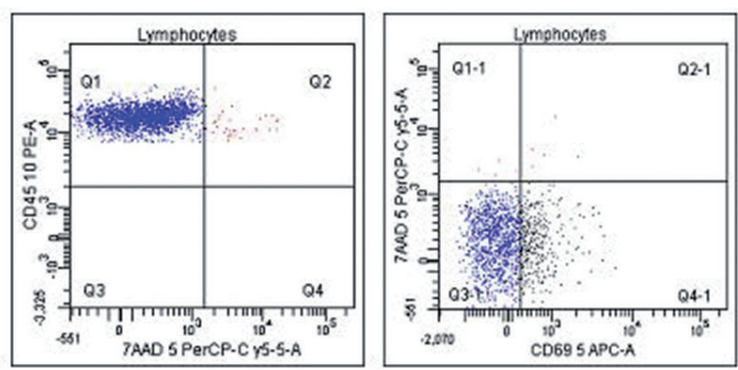

Irradiated ( $R$
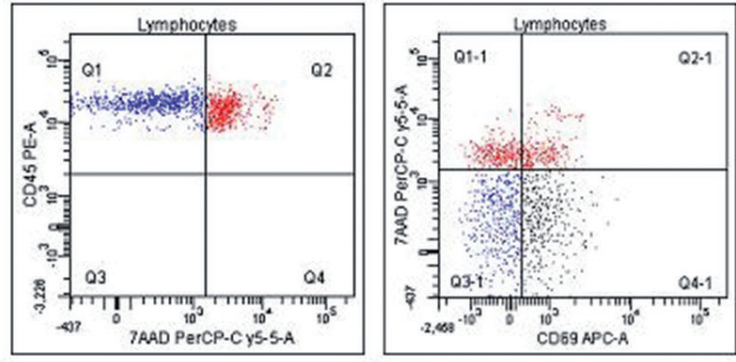

Miras of PRT System
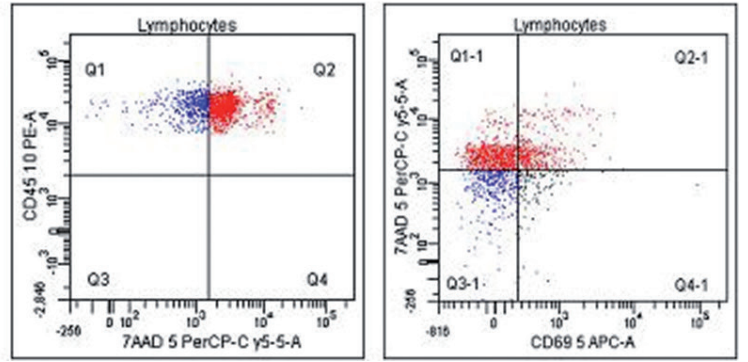

Fig. 1. Flow cytometry analysis. A - principle of the cytometry analysis. Cytogram "a" presents the method of separation of lymphocytes with low SSC and high intensity of CD45 expression (blue gate). Cytogram "b" presents 7-AAD expression on control lymphocytes on storage day 1. Viable cells are located in Q1 and nonviable cells in Q2 (CD45+7-AAD+) (red gate). 7-AAD expression in control samples on day 1 was the reference for samples on all consecutive days. Cytogram "C" presents CD69 expression on CD45+7-AAD- lymphocytes. Activated CD69+ lymphocytes are located in the Q4-1 region. CD69-positive cell marker was based on the expression of the isotope control. B - 7-AAD and CD69 expression on lymphocytes in controls following irradiation and Mirasol ${ }^{\circledR}$ PRT System on day 6 of storage ples. During the 6 days of storage, the number of viable cells decreased to $28 \%(\mathrm{p}=0.001)$ and to $65 \%(\mathrm{p}=0.004)$ following Mirasol ${ }^{\circledR}$ PRT System treatment and gamma irradiation, respectively. The percentage of viable cells in the $M$ treated samples was also significantly lower than in the R samples ( $\mathrm{p}=0.001$ ) (Fig. 1B, Fig. 2).

\section{Lymphocyte activation: CD69 expression}

CD69 expression and the mean fluorescent intensity of CD69 were determined only for viable cells 7AAD $(-)$ (Fig. 1B). Lymphocyte activation, presented in the form of a percentage of CD69 expression, ranged from 20\% to $40 \%$ of the CD69-positive cells throughout the storage period (day 1, 3 and 6). The Mean Fluorescent Intensity (MFI) of CD69 was the lowest in the $M$ treated samples (Table 1). Due to the small number of experiments $(n=7)$, the comparison of average values was less representative and so CD69 expression was monitored as a ratio of treated/control values in individual PC units. In relation to the controls, the values below 1 represent a decrease in CD69 expression and values above 1 represent an increase (Fig. 3). Imme-
Table 1. CD69 mean fluorescence intensity ( $\left.{ }^{*} \mathrm{MFI}\right)$ in samples during 6-day storage

\begin{tabular}{|l|c|c|}
\hline Time point & Sample & CD69 MFF* Mean \pm SD \\
\hline Day 1 & C & $502 \pm 141$ \\
& R & $513 \pm 135$ \\
Day 3 & M & $379 \pm 107$ \\
& C & $646 \pm 153$ \\
Day 6 & R & $621 \pm 148$ \\
& M & $383 \pm 126$ \\
& C & $675 \pm 167$ \\
\hline
\end{tabular}

diately after Mirasol ${ }^{\circledR}$ PRT System treatment, a statistically significant (1.4-fold) decrease in the number of CD69-positive lymphocytes was observed as compared to untreated controls; the tendency was reported for the entire storage period ( $\mathrm{p}<0.001$ ). On storage day 3, no changes in CD69 expression were observed in the R PCs as compared to the C PCs. On day 6 however, there occurred a slight, though statistically significant, increase in the number of CD69positive lymphocytes ( $\mathrm{p}<0.05)$. Moreover, CD69 expression on the lymphocytes in the M treated PCs was sig- 
\% 7-AAD(-) Control (C) $\quad$ Irradiated (R) $\square \quad$ Mirasol ${ }^{\circledR}$ PRT System (M)

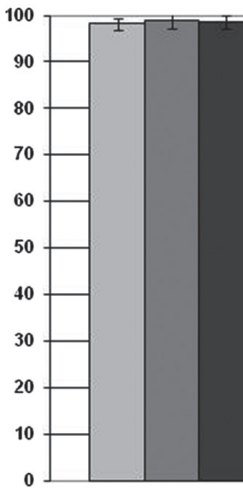

day 1

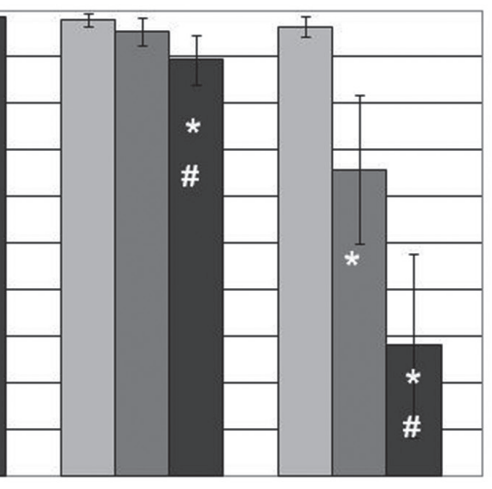

day 3

day 6

Fig. 2. Viability of lymphocytes during 6 days of storage measured by percentage of non-permeable viable cells 7-AAD(-) in PCs subjected to irradiation and Mirasol ${ }^{\circledR}$ PRT System. $\left(^{*}\right)$ indicates a statistically significant difference between study samples $(R, M)$ and control samples $(C)$. (\#) indicates a statistically significant difference between $\mathrm{R}$ samples and M samples. Results are shown as mean and standard deviation

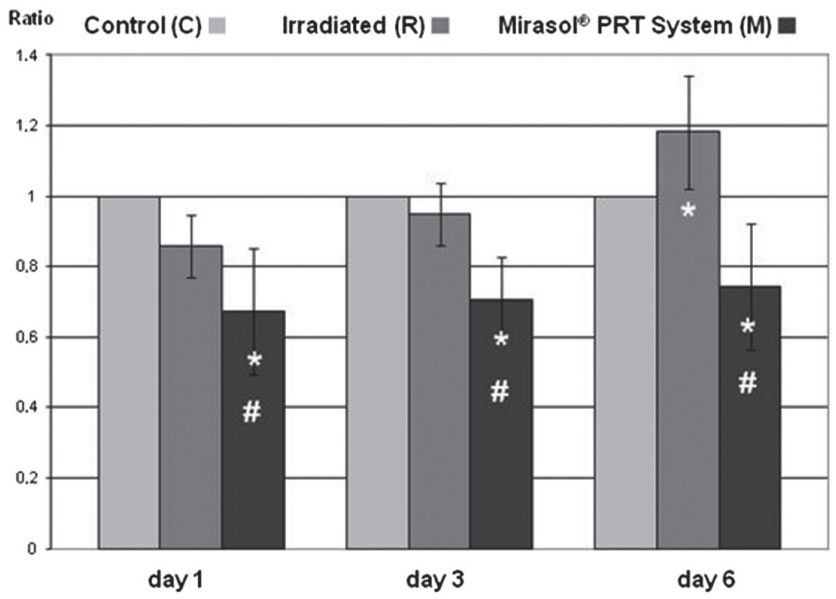

Fig. 3. Changes in lymphocyte CD69 expression on days 1, 3 and 6 in $\mathrm{R}$ samples and $\mathrm{M}$ samples as compared to $\mathrm{C}$ samples. Coefficient values indicate the increase or decrease of parameters calculated as ratio: \%CD69+ in the treated/control samples. A separate analysis was performed for each study experiment $(n=7)$. $\left(^{*}\right)$ indicates a statistically significant difference between $\mathrm{R}$ and $\mathrm{M}$ samples and $\mathrm{C}$ samples. (\#) indicates a statistically significant difference between $R$ samples and M samples. Results are shown as mean and standard deviation

nificantly lower than on lymphocytes in the R PCs (day 1: $\mathrm{p}<0.01$, day 3: $\mathrm{p}<0.01$ and day 6: $\mathrm{p}<0.001$ ) (Fig. 1B, Fig. 3). The values of CD69 expression in the $C, R$ and $M$ samples on storage days 3 and 6 indicated that the percentage of activated lymphocytes in the control samples did not change significantly during storage compared to the values obtained on day 1 . A statistically significant increase in CD69 expression in the stored R PC samples was observed; 1.3-fold and 1.5-fold on days 3 and 6, respectively. In the $M$ treated samples, no statistically significant increase in CD69 expression was observed (Fig. 4).

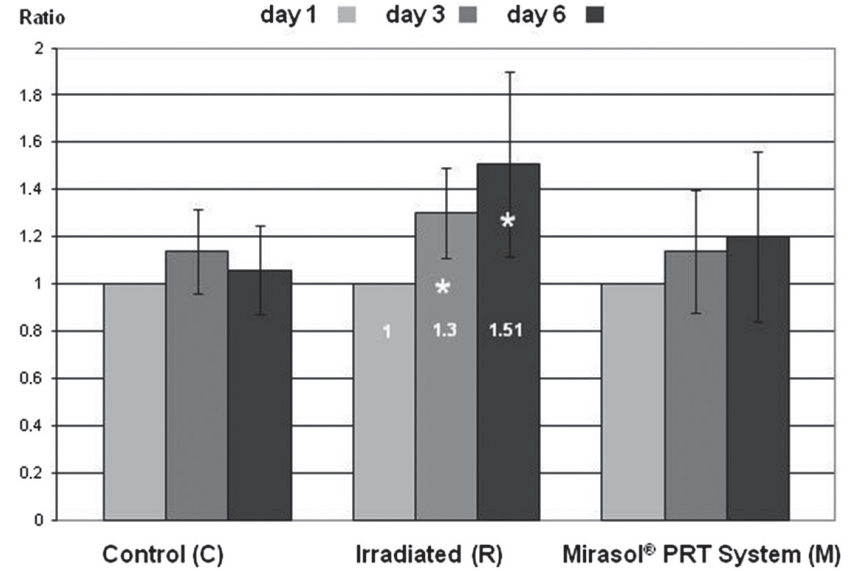

Fig. 4. Changes in lymphocyte CD69 expression after 6 days of storage in C, $\mathrm{R}$ and $\mathrm{M}$ samples. The coefficient value reflecting the increase or decrease of parameters was calculated as ratio: \%CD69+ after 3 days and 6 days of storage/value on day 1. A separate analysis was made for each consecutive experiment series $(n=7)$. $\left(^{*}\right)$ indicates a statistically significant difference compared to day 1. Results are shown as mean and standard deviation

\section{Discussion}

TA-GvHD is caused by viable lymphocytes in blood components. It is diagnosed in severely immunocompromised patients as well as in patients with some degree of HLA homozygosity with the donor of the blood component. ${ }^{1}$ During storage of transfusion-dedicated PCs, numerous soluble factors are released by residual leukocytes and platelets, including pro-inflammatory cytokines and chemokines. The concentration of these soluble factors varies depending on the method of $\mathrm{PC}$ preparation and leukocyte content in the blood components. ${ }^{18}$

To date, gamma irradiation has been the main technology used for inactivation of residual lymphocytes in transfusion-dedicated blood components and for TAGvHD prevention. Irradiation technology, however, is not effective for pathogen reduction. To reduce the risk of pathogen transmission, many countries have therefore implemented pathogen reduction technologies, which have also been found effective for lymphocyte inactivation in blood components dedicated for clinical use. In the case of platelets, these technologies are the Mirasol ${ }^{\circledR}$ PRT System and Intercept ${ }^{\mathrm{TM}}$ Blood System, which are also effective for $\mathrm{T}$ lymphocyte inactivation. ${ }^{6,7}$

Literature reviews confirm that pathogen reduction technology methods (Mirasol ${ }^{\circledR}$ PRT System and Intercept $^{\mathrm{TM}}$ Blood System) have proved effective for leukocyte inactivation in cellular blood components. This implies that pathogen inactivated PCs (with either the Mirasol ${ }^{\circledR}$ PRT System or Intercept ${ }^{\mathrm{TM}}$ Blood System) are as safe for patients at risk of TA-GvHD as irradiated PCs. ${ }^{11-13}$

This study focused on leukocyte inactivation with the Mirasol ${ }^{\circledR}$ PRT System because this pathogen-reduction system is in routine use in most Polish blood transfusion centers. Another reason for the choice of the Mirasol ${ }^{\circledR}$ 
PRT System is that, to date, the Intercept ${ }^{\mathrm{TM}}$ Blood System has been far better described in literature than Mirasol.

In the study, lymphocyte viability and the CD69 early activation marker were the two parameters monitored on unstimulated lymphocytes in non-leukoreduced PCs during 6 days of storage with the purpose of evaluation of the effectiveness of both Mirasol ${ }^{\circledR}$ PRT System treatment and Gammacell 3000 Elan irradiation. The Mirasol ${ }^{\circledR}$ PRT System was found even more effective for lymphocyte inactivation than gamma irradiation. After 6 days of storage, a significant decrease in the number of viable lymphocytes in the M group of PCs was reported (from $99 \%$ to $28 \%$ ) whereas the number of viable lymphocytes in the $\mathrm{C}$ group of PCs remained at the same level (> 95\%) throughout the 6 days of storage. The decrease in the number of viable lymphocytes in the R group of PCs was smaller (from 99\% to 65\%). Similar results were reported by Jackman et al., who used amine reactive dye; only about $5 \%$ of viable cells were found in the M treated PCs (already on storage day 3) while about $50 \%$ were reported in the $\mathrm{R} \mathrm{PCs} .{ }^{19}$

According to Diacovo et al., an interaction occurs between lymphocytes and activated platelets that results in lymphocyte activation and higher CD69 expression. ${ }^{20}$ Pócsik et al. reported an increase in the expression of the interleukin-2 receptor, CD26, activation-inducer molecule (AIM, CD69) and transferring receptors (CD71) 3 days after allotransfusion of platelets, which indicated an important functional molecule expression on lymphocytes activated by allogenic platelets. ${ }^{21}$ Our study results comply with this observation; after one-day storage, approximately $35 \%$ of untreated lymphocytes were found CD69-positive and the level of activated lymphocytes remained the same during the entire storage period. A similar percentage of activated lymphocytes was observed in the R PCs, but the percentage and intensity of CD69 expression increased 1.2-fold on day 6 of storage as compared to the $\mathrm{C}$ samples. In the M PCs, however, the activated lymphocyte count decreased by about 1.4-fold on day 1 as compared to the $C$ samples. The level was maintained throughout the whole storage period.

Fast et al. demonstrated that the Mirasol ${ }^{\circledR}$ PRT System inhibits the proliferative capacity of $\mathrm{T}$ lymphocytes. This was confirmed by a complete lack of CD69 expression in the $\mathrm{M}$ treated $\mathrm{T}$ cells $(1.7 \pm 1.3 \%)$ in comparison to CD69 antigen expression in the control cells: $64.4 \pm 15.6 \%$. CD69 antigen expression is the measure of T lymphocyte proliferative capacity. ${ }^{9}$

Unlike gamma irradiation, the Mirasol ${ }^{\circledR}$ PRT System is reported in literature analyses as effective for the prevention of antigen presentation and therefore also cytokine synthesis. The Mirasol ${ }^{\circledR}$ PRT System may be responsible for reduced production of cytokines which are involved in non-haemolytic transfusion reactions and production of the alloantibodies involved in adverse transfusion-related reactions. ${ }^{21}$ The Mirasol ${ }^{\circledR}$ PRT System may also be used for inactivation of leukocytes in blood components due to inhibition of nucleic acid replication. ${ }^{11}$

The in vitro results for the three PC study groups (C, R and M) suggest that the Mirasol ${ }^{\circledR}$ PRT System is as effective as gamma irradiation due to its capacity to inactivate donor leukocytes.

\section{References}

1. Dwyre DM, Holland PV. Transfusion-associated graft-versus-host disease. Vox Sang. 2008;95:85-93.

2. Pogłód R. Transfusion-associated - Graft versus Host Disease (TAGvHD). Acta Haematologica Polonica. 2009;40(2):425-434.

3. Annual SHOT Report 2015. http://www.shotuk.org/wp-content/ uploads/SHOT-2015-Annual-Report-Web-Edition-Final-bookmarked-1.pdf. Accessed September 14, 2016.

4. Moroff G, Luban NLC. The irradiation of blood and blood components to prevent graft-versus-host disease: Technical issues and guidelines. Transfus Med Rev. 1997;11:15-26.

5. Mintz PD, Wehrli G. Irradiation eradication and pathogen reduction. Ceasing cesium irradiation of blood products. Bone Marrow Transplant. 2009;44:205-211.

6. Fast LD, DiLeone G, Marschner S. Inactivation of human white blood cells in platelet products after pathogen reduction technology treatment in comparison to gamma irradiation. Transfusion. 2011;51:1397-1404.

7. Knutson F, Osselaer J, Pierelli L, et al. A prospective, active haemovigilance study with combined cohort analysis of 19175 transfusions of platelet components prepared with amotosalen-UVA photochemical treatment. Vox Sang. 2015;109:343-352.

8. Luban NLC, Drothler D, Moroff G, Quinones R. Irradiation of platelet components: inhibition of lymphocyte proliferation assessed by limiting-dilution analysis. Transfusion. 2000;40:348-352.

9. Fast LD, DiLeone G, Li J, Goodrich R. Functional inactivation of white blood cells by Mirasol treatment. Transfusion. 2006;46:642-648.

10. Reddy M, Eirikis E, Davis C, Davis HM, Prabhakar U. Comparative analysis of lymphocyte activation marker expression and cytokine secretion profile in stimulated human peripheral blood mononuclear cell cultures: An in vitro model to monitor cellular immune function. J Immunol Methods. 2004;293:127-142.

11. Marschner S, Fast LD, Baldwin III WM, Slichter SJ, Goodrich RP. White blood cell inactivation after treatment with riboflavin and ultraviolet light. Transfusion. 2010;50:2489-2498.

12. Cazenave JP, Follea G, Bardiaux L, et al. A randomized controlled clinical trial evaluating the performance and safety of platelets treated with MIRASOL pathogen reduction technology. Transfusion. 2010;50:2362-2375.

13. Fast LD, DiLeone G, Cardarelli G, Li J, Goodrich R. Mirasol PRT treatment of donor white blood cells prevents the development of xenogeneic graft-versus-host disease in Rag $2^{-1-} \mathrm{Yc}^{-1-}$ double knockout mice. Transfusion. 2006;46:1553-1560.

14. Muylle L, Peetermans ME. Effect of prestorage leukocyte removal on the cytokine levels in stored platelet concentrates. Vox Sang. 1994;66:14-17.

15. Antoniewicz-Papis J, Dąbrowska A. Preparation of blood and blood components. In: Łętowska M, ed. Medical standards for collection, preparation and distribution of blood and blood components. $3^{\text {rd }}$ ed. Warszawa: Institute of Hematology and Transfusion Medicine; 2014:242-249.

16. Lachert E, Kubis J, Dąbrowska A. Quality Assurance System in Blood Transfusion Service. In: Łętowska M, ed. Medical standards for collection, preparation and distribution of blood and blood components. $3^{\text {rd }}$ ed. Warszawa: Institute of Hematology and Transfusion Medicine; 2014:91-92.

17. Ruane PH, Edrich R, Gampp D, Keil SD, Leonard RL, Goodrich RP. Photochemical inactivation of selected viruses and bacteria In platelet concentrates using riboflavin and light. Transfusion. 2004; 44:877-885.

18. Picker SM, Steisel A, Gathof BS. Evaluation of white blood cell- and platelet-derived cytokine accumulation in MIRASOL-PRT-Treated platelets. Transfusion Medicine and Hemotherapy. 2009;36:114-120. 
19. Jackman RP, Heitman JW, Marschner S, Goodrich RP, Norris PJ. Understanding loss of donor white blood cell immunogenicity after pathogen reduction: Mechanisms of action in ultraviolet illumination and riboflavin treatment. Transfusion. 2009;49:2686-2699.
20. Diacovo TG, Catalina MD, Siegelman MH, von Andrian UH. Circulating activated platelets reconstitute lymphocyte homing and immunity in L-selectin-deficient mice. J Exp Med. 1998;187;197-204.

21. Pócsik É, Mihalik R, Gyódi É, et al. Activation of lymphocytes after platelet allotransfusion possessing only class I MHC product. Clin Exp Immunol. 1990;82:102-107. 